Supporting Information

\title{
High-Performance n-Type Ge-Free Silicon Thermoelectric Material from Silicon Waste
}

Zhenhui Liu ${ }^{\mathrm{a}}$, Qihao Zhang ${ }^{\mathrm{a} *}$, Ulrike Wolff ${ }^{\mathrm{a}}$, Christian G. F. Blum ${ }^{\mathrm{a}}$, Ran He $\mathrm{He}^{\mathrm{a}}$, Amin Bahrami $^{\mathrm{a}}$, Maximilian Beier-Ardizzon ${ }^{\mathrm{c}}$, Christian Reimann $^{\mathrm{c}}$, Jochen Friedrich ${ }^{\mathrm{c}}$, Heiko

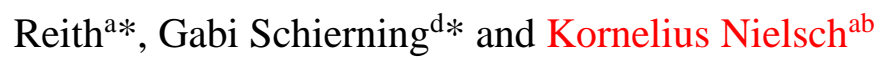

${ }^{a}$ Leibniz Institute for Solid State and Materials Research Dresden (IFW Dresden), 01069, Dresden, Germany.

${ }^{b}$ Institute of Materials Science, Dresden University of Technology (TU Dresden), 01062, Dresden, Germany

${ }^{c}$ Fraunhofer Institute for Integrated Systems and Device Technology, 91058, Erlangen, Germany

${ }^{d}$ Faculty of Physics, Bielefeld University, 33615, Bielefeld, Germany

*E-mail: q.zhang@ifw-dresden.de; h.reith@ifw-dresden.de;

gschierning@physik.uni-bielefeld.de 


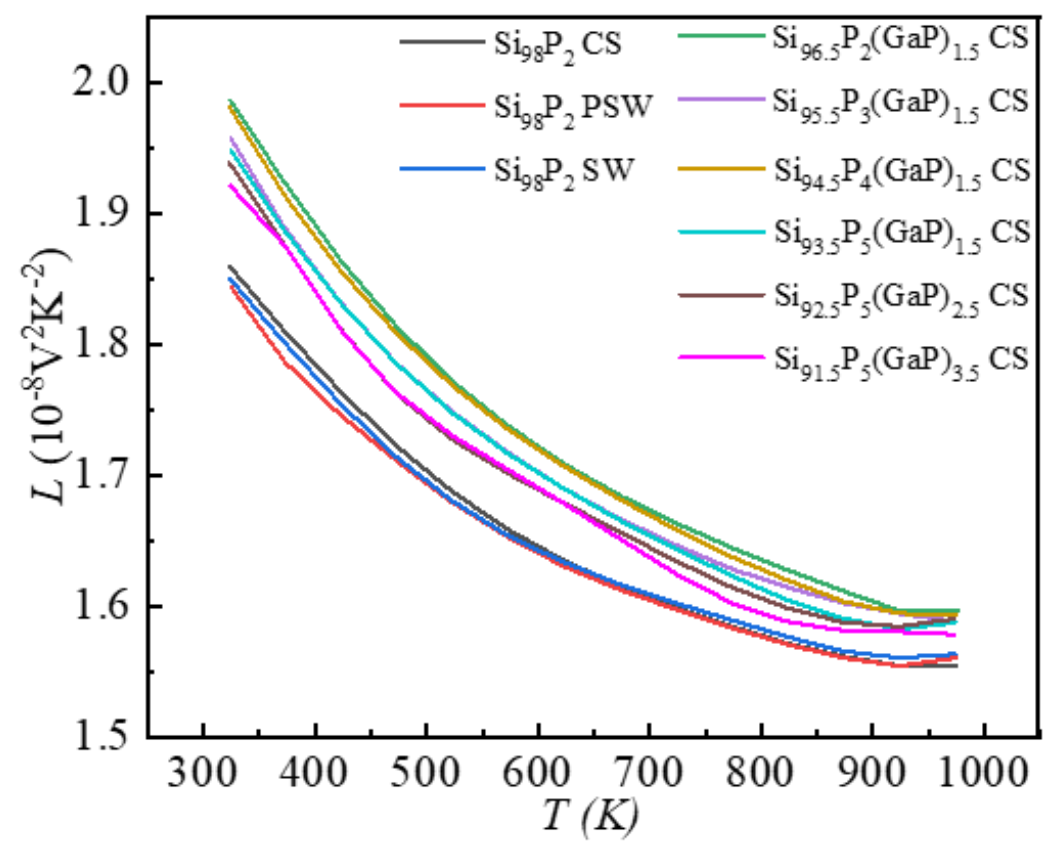

Figure S1. Temperature-dependent Lorenz number calculated by using single parabolic band (SPB) approximation.

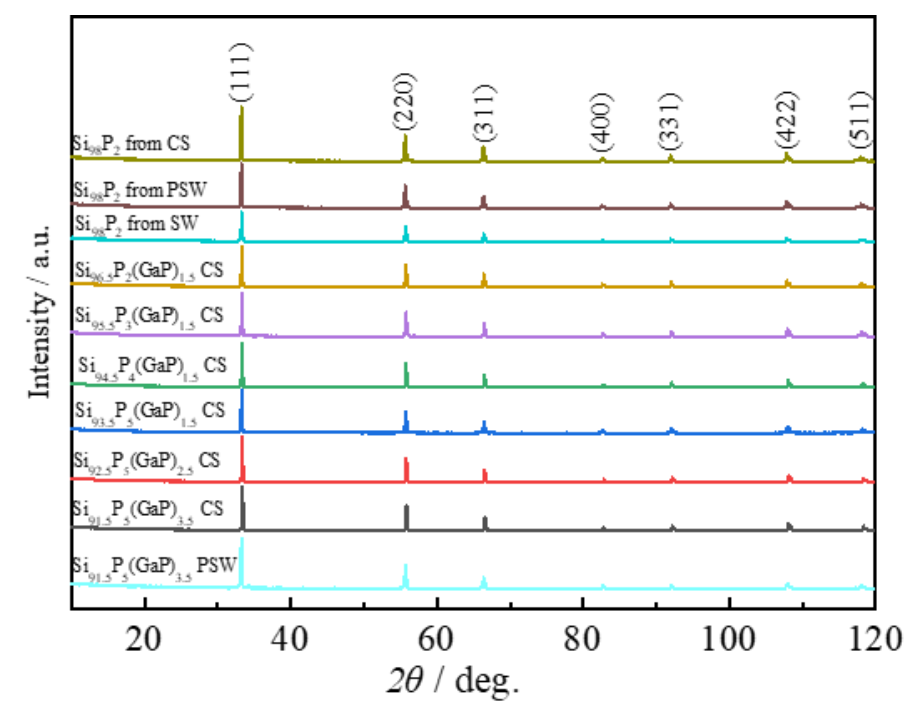

Figure S2. XRD patterns of samples made from commercial silicon (CS), purified silicon waste (PSW), and silicon waste (SW). 


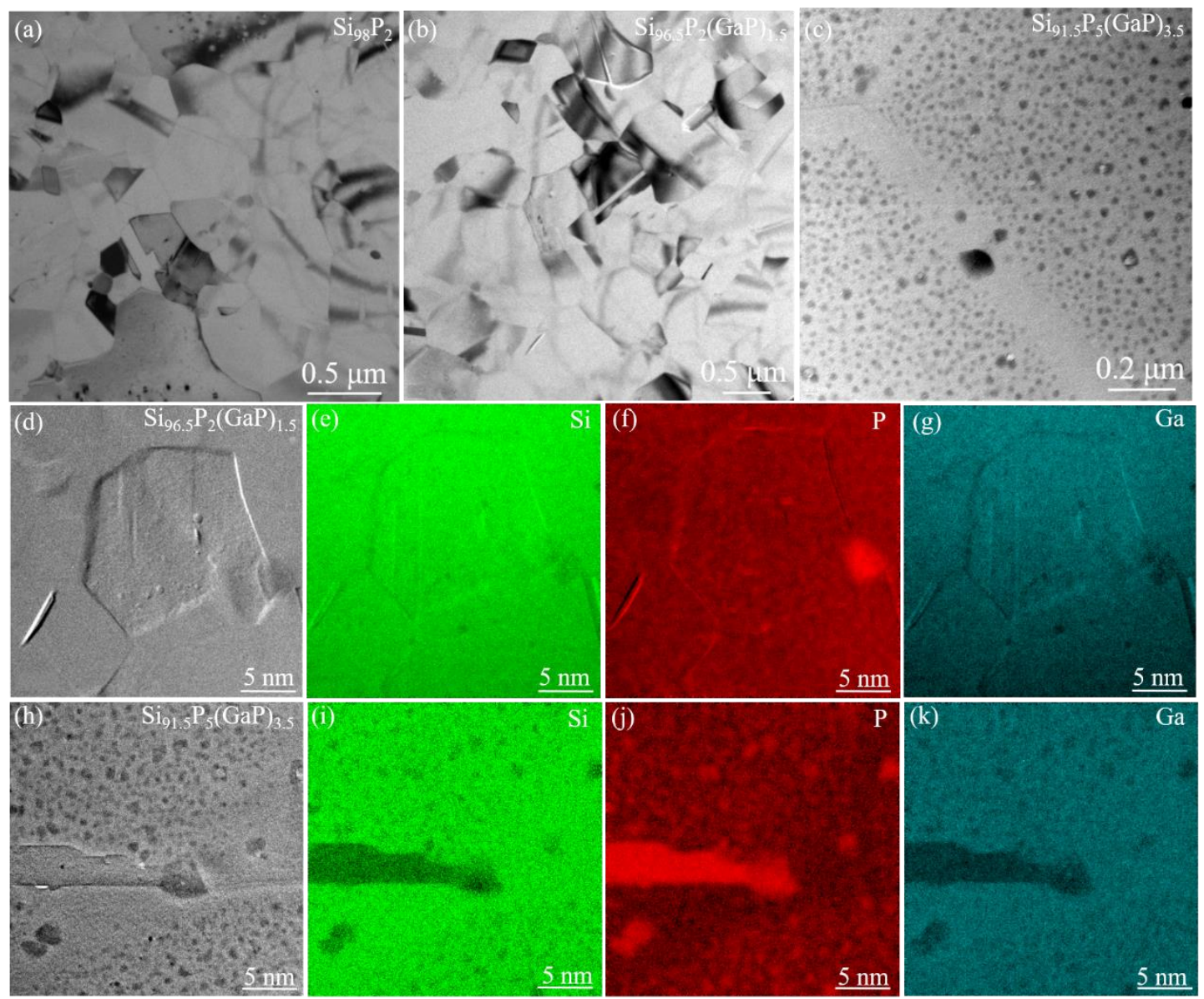

Figure S3. TEM characterization of selected $\mathrm{Si}_{1-\mathrm{x}-\mathrm{y}} \mathrm{P}_{\mathrm{x}}(\mathrm{GaP})_{\mathrm{y}}$ samples: low-magnification TEM image of a) $\mathrm{Si}_{98} \mathrm{P}_{2}$, b) $\mathrm{Si}_{96.5} \mathrm{P}_{2}(\mathrm{GaP})_{1.5}$ and c) $\mathrm{Si}_{91.5} \mathrm{P}_{5}(\mathrm{GaP})_{3.5}$, high-magnification TEM image of d) $\mathrm{Si}_{96.5} \mathrm{P}_{2}(\mathrm{GaP})_{1.5}$ and corresponding elemental mapping of e) $\mathrm{Si}, \mathrm{f}$ ) $\mathrm{P}$, and $\mathrm{g}$ ) $\mathrm{Ga}$ and high-magnification TEM image of $\mathrm{h}$ ) $\mathrm{Si}_{91.5} \mathrm{P}_{5}(\mathrm{GaP})_{3.5}$ and corresponding elemental mapping of i) $\mathrm{Si}, \mathrm{j}$ ) $\mathrm{P}$ and k) $\mathrm{Ga}$. 

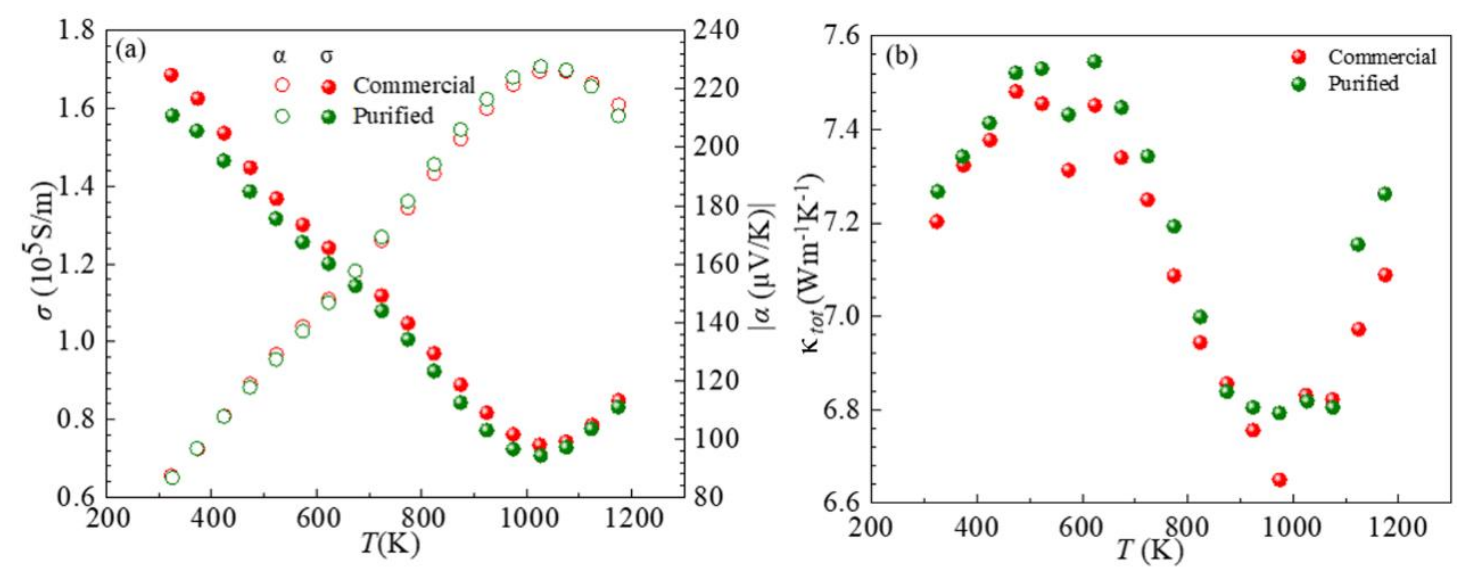

Figure S4. TE properties of $\mathrm{Si}_{91.5} \mathrm{P}_{5}(\mathrm{GaP})_{3.5}$ samples made from different Si sources. a) Temperature-dependent electrical conductivity $\sigma$ and absolute Seebeck coefficient $|\alpha|$. b) Temperature-dependent total thermal conductivity.

Details of lattice thermal conductivity calculation based on Callaway model ${ }^{1,2}$ :

$$
\kappa_{L}=\frac{k_{B}}{2 \pi^{2} v}\left(\frac{k_{B} T}{\hbar}\right)^{3} \int_{0}^{\theta_{D} / T} \frac{x^{4} e^{x}}{\tau_{C}^{-1}\left(e^{x}-1\right)^{2}} d x
$$

where $\mathrm{x}=\hbar \omega /\left(\mathrm{k}_{\mathrm{B}} T\right), \mathrm{k}_{\mathrm{B}}, \omega, \hbar, \theta_{\mathrm{D}}, \mathrm{v}$ and $\tau_{\mathrm{C}}$ are the reduced phonon frequency, Boltzmann constant, phonon frequency, reduced Planck constant, Debye temperature, sound velocity and phonon-scattering relaxation time, respectively. $\theta_{\mathrm{D}}$ and $\mathrm{v}$ of silicon can be found in previous research. ${ }^{3}$

$$
\tau_{C}^{-1}=A \omega^{4}+B \omega^{2} T \exp \left(-\theta_{D} / 3 T\right)+C \omega^{2}+\frac{v}{d}
$$

$\mathrm{A}, \mathrm{B}, \mathrm{C}$ and $\mathrm{v} / \mathrm{d}$, which are listed in Table $\mathrm{S} 1$, are pre-factors for point defect scattering, phonon-phonon Umklapp scattering, electron-phonon scattering and grain boundary scattering, respectively, and $\mathrm{d}$ is the grain size. 
Table S1. Calculated pre-factors for different scattering mechanisms and experimental carrier concentration for selected samples.

\begin{tabular}{llllll}
\hline Composition & $\mathrm{v} / \mathrm{d}\left(10^{9} \mathrm{~s}^{-1}\right)$ & $\mathrm{A}\left(10^{-45} \mathrm{~s}^{3}\right)$ & $\mathrm{B}\left(10^{-20} \mathrm{~s} / \mathrm{K}\right)$ & $\mathrm{C}\left(10^{-17} \mathrm{~s}\right)$ & $\mathrm{n}_{\mathrm{H}}\left(10^{20} \mathrm{~cm}^{-3}\right)$ \\
\hline $\mathrm{Si}_{98} \mathrm{P}_{2}$ & 11.8 & -- & 37 & 40 & 2.6 \\
$\mathrm{Si}_{9.5} \mathrm{P}_{5}(\mathrm{GaP})_{3.5}$ & 11.8 & 190 & 37 & 56.1 & 3.35 \\
\hline
\end{tabular}

Details for determining the pre-factors:

(1) d was obtained through the distribution of grain size from TEM images, and the average grain size of the samples in this work is around $500 \mathrm{~nm}$.

(2) To determine the value of B: For single crystal silicon, only phonon-phonon Umklapp scattering is considered, so B is acquired by fitting with known $\kappa_{L}$ from previous research. ${ }^{3,4}$

(3) To determine the value of C: For the pre-factors of point defect scattering, it is mainly determined by the fluctuation of atomic mass and radius between impurity atom and host lattice. ${ }^{5} \mathrm{P}$ and $\mathrm{Si}$ are neighboring elements, and their mass and radius are comparable. Hence, for $\mathrm{Si}_{98} \mathrm{P}_{2}$, the contribution of point defect scattering for the reduction of lattice thermal conductivity is negligible, which has been also proved in former work. ${ }^{3}$ Therefore, for the $\mathrm{Si}_{98} \mathrm{P}_{2}$ sample, the point defect scattering is not considered, and only phonon-phonon Umklapp scattering, electron-phonon and boundary scattering are considered. By fitting the experimental $\kappa_{L}$, the pre-factor of electron-phonon scattering for $\mathrm{Si}_{98} \mathrm{P}_{2}\left(\mathrm{C}_{1}\right)$ can be determined after B is obtained. $C$ is proportional to $n_{H}^{4 / 3}$ if we assume the other pre-factors are independent of carrier 
concentration $^{6}$, therefore, the pre-factor of electron-phonon scattering for $\mathrm{Si}_{96.5} \mathrm{P}_{2}(\mathrm{GaP})_{1.5}\left(\mathrm{C}_{2}\right)$ and $\mathrm{Si}_{91.5} \mathrm{P}_{5}(\mathrm{GaP})_{3.5}\left(\mathrm{C}_{3}\right)$ can be determined in this way.

(4) To determine the value of A: For $\mathrm{Si}_{96.5} \mathrm{P}_{2}(\mathrm{GaP})_{1.5}$ sample, d, B and C are obtained in step 1, 2 and 3. With known $\kappa_{L}$, the pre-factor of point defect scattering of $\mathrm{Si}_{96.5} \mathrm{P}_{2}(\mathrm{GaP})_{1.5}\left(\mathrm{~A}_{2}\right)$ is acquired through fitting. For $\mathrm{Si}_{91.5} \mathrm{P}_{5}(\mathrm{GaP})_{3.5}$ sample, from TEM analysis, when more GaP was added, Ga did not distribute as homogeneously as that of the $\mathrm{Si}_{96.5} \mathrm{P}_{2}(\mathrm{GaP})_{1.5}$ sample, and enrichment of $\mathrm{Ga}$ is observed. For this sample, therefore, just 1.5 at $\% \mathrm{Ga}$ is believed to be dissolved, and the pre-factor of point defect scattering of $\mathrm{Si}_{91.5} \mathrm{P}_{5}(\mathrm{GaP})_{3.5}\left(\mathrm{~A}_{3}\right)$ is equal to $\mathrm{A}_{2}$.

\section{References}

[1] Lo, S. H.; He, J.; Biswas, K.; Kanatzidis, M. G.; Dravid, V. P. Phonon Scattering and Thermal Conductivity in p-type Nanostructured PbTe-BaTe Bulk Thermoelectric Materials. Adv. Funct. Mater. 2012, 22, 5175-5184.

[2] Wan, S.; Qiu, P.; Huang, X.; Song, Q.; Bai, S.; Shi, X.; Chen, L. Synthesis and Thermoelectric Properties of Charge-Compensated $\mathrm{S}_{\mathrm{y}} \mathrm{Pd}_{\mathrm{x}} \mathrm{Co}_{-\mathrm{x}} \mathrm{Sb}_{12}$ Skutterudites. ACS Appl. Mater. Interfaces 2018, 10, 625-634.

[3] Zhu, T.; Yu, G.; Xu, J.; Wu, H.; Fu, C.; Liu, X.; Zhao, X. The Role of Electron-Phonon Interaction in Heavily Doped Fine-Grained Bulk Silicons as Thermoelectric Materials. Adv. Electron. Mater. 2016, 2, 1600171.

[4] Glassbrenner, C. J.; Slack, G. A. Thermal Conductivity of Silicon and Germanium from $3 \mathrm{~K}$ to the Melting Point. Phys. Rev. 1964, 134, A1058-A1069. 
[5] Yang, J.; Meisner, G. P.; Chen, L. Strain Field Fluctuation Effects on Lattice Thermal Conductivity of ZrNiSn-based Thermoelectric Compounds. Appl. Phys. Lett., 2004, 85, 1140-1142.

[6] Shi, X.; Pei, Y.; Snyder, G. J.; Chen, L. Optimized Thermoelectric Properties of $\mathrm{Mo}_{3} \mathrm{Sb}_{7-} \mathrm{Te}_{\mathrm{x}}$ with Significant Phonon Scattering by Electrons. Energy Environ. Sci., 2011, 4, 4086-4095. 